\title{
Matrix elements of electroweak penguin operators and the large $-N_{C}$ expansion
}

\section{Marc Knecht*}

Centre de Physique Théorique, CNRS-Luminy Case 907, F-13288 Marseille Cedex 9,

France

E-mail: 'knecht@cpt.univ-mrs.fri

ABSTRACT: We review the recent determination of the matrix elements of the electroweak penguin operators $Q_{7}$ and $Q_{8}$ at order $\mathcal{O}\left(p^{0}\right)$ and in the framework of the large- $N_{C}$ expansion of QCD. We discuss several issues, like the scale and scheme dependence, and compare our results to those obtained through dispersion relations and sum rules, or from numerical simulations on the lattice.

\section{Introduction}

At low energies, the effective Hamiltonian of $\Delta S=1$ weak hadronic transitions is described in terms of a set of four-quark operators $Q_{i}(\mu, \kappa)$, modulated by Wilson coefficients $C_{i}(\mu, \kappa)$,

$$
\mathcal{H}^{\Delta S=1}=\frac{G_{F}}{\sqrt{2}} V_{u d} V_{u s}^{*} \sum_{i} C_{i}(\mu, \kappa) Q_{i}(\mu, \kappa) .
$$

The latter contain the information from the short distances, and can be computed perturbatively. The perturbative contributions need however to be renormalized, so that the coefficients $C_{i}(\mu, \kappa)$ depend on the renormalization scale $\mu$ introduced by the minimal subtraction in dimensional regularization, as well as on the scheme used in order to define the $\gamma_{5}$ matrix in $D$ dimensions, the basis of evanescent operators, and the like [iin. This is indicated by the the presence of the second argument, $\kappa$. The long-distance physics is contained in the hadronic matrix elements of the four-quark operators $Q_{i}(\mu, \kappa)$, which depend likewise on the renormalization scale $\mu$ and on the scheme $\kappa$ involved in the computation of the Wilson coefficients. Physical quantities like hadronic matrix elements of $\mathcal{H}^{\Delta S=1}$ should depend neither on the choice of the factorization scale $\mu$, nor on the scheme $\kappa$. This requirement puts a stringent constraint on any theoretical or phenomenological approach aiming at evaluating the matrix elements of the four-quark operators $Q_{i}(\mu, \kappa)$.

${ }^{*}$ Speaker. 
The present contribution, based on the work published in "i2i], is devoted to the electroweak penguins $Q_{7}$ and $Q_{8}$. We shall consider the $\Delta I=3 / 2$ components, $Q_{7}^{(3 / 2)}$ and $Q_{8}^{(3 / 2)}$, of these operators,

$$
\begin{aligned}
& Q_{7}^{(3 / 2)}=2\left(\overline{s_{L}} \gamma_{\mu} d_{L}\right)\left[\left(\overline{u_{R}} \gamma^{\mu} u_{R}\right)-\left(\overline{d_{R}} \gamma^{\mu} d_{R}\right)\right]+2\left(\overline{s_{L}} \gamma_{\mu} u_{L}\right)\left(\overline{u_{R}} \gamma^{\mu} d_{R}\right) \\
& Q_{8}^{(3 / 2)}=-4\left(\overline{s_{L}} u_{R}\right)\left(\overline{u_{R}} d_{L}\right)+4\left(\overline{s_{L}} d_{R}\right)\left[\left(\overline{d_{R}} d_{L}\right)-\left(\overline{u_{R}} u_{L}\right)\right]
\end{aligned}
$$

and restrict ourselves to the chiral limit, where the $K \rightarrow \pi \pi$ and $K \rightarrow \pi$ matrix elements are given as

$$
\begin{aligned}
\left\langle(\pi \pi)_{2}\left|Q_{7}^{(3 / 2)}\right| K^{0}\right\rangle & =-\frac{1}{F_{0}}\left\langle\pi^{+}\left|Q_{7}^{(3 / 2)}\right| K^{+}\right\rangle=-\frac{4}{F_{0}^{3}}\left\langle O_{1}\right\rangle \\
\left\langle(\pi \pi)_{2}\left|Q_{8}^{(3 / 2)}\right| K^{0}\right\rangle & =-\frac{1}{F_{0}}\left\langle\pi^{+}\left|Q_{8}^{(3 / 2)}\right| K^{+}\right\rangle=\frac{8}{F_{0}^{3}}\left\langle O_{2}\right\rangle,
\end{aligned}
$$

with $\left\langle O_{1}\right\rangle=\left\langle 0\left|\left(\overline{s_{L}} \gamma_{\mu} d_{L}\right)\left(\overline{d_{R}} \gamma^{\mu} s_{R}\right)\right| 0\right\rangle,\left\langle O_{2}\right\rangle=\left\langle 0\left|\left(\overline{s_{L}} s_{R}\right)\left(\overline{d_{R}} d_{L}\right)\right| 0\right\rangle$. Note that $O_{1}$ is a current-current operator, whereas $O_{2}$ is a density-density operator. Their vacuum expectation values have therefore very different behaviours in the $1 / N_{C}$ expansion. Thus, $\left\langle O_{1}\right\rangle$ has no factorized contribution, and behaves as $\mathcal{O}\left(N_{C}\right),\left\langle O_{1}\right\rangle=0+\left.\left\langle O_{1}\right\rangle\right|_{\mathcal{O}\left(N_{C}\right)}+\mathcal{O}\left(N_{C}^{0}\right)$. On the other hand, the LO contribution to $\left\langle O_{2}\right\rangle$ is a $\mathcal{O}\left(N_{C}^{2}\right)$ factorized piece, while the NLO correction vanishes, $\left\langle O_{2}\right\rangle=\left.\left\langle O_{1}\right\rangle\right|_{\mathcal{O}\left(N_{C}^{2}\right)}+0+\left.\left\langle O_{2}\right\rangle\right|_{\mathcal{O}\left(N_{C}^{0}\right)}+\mathcal{O}\left(N_{C}^{-1}\right)$.

\section{Evaluation of $\left\langle O_{1}\right\rangle$}

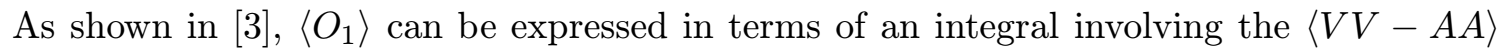

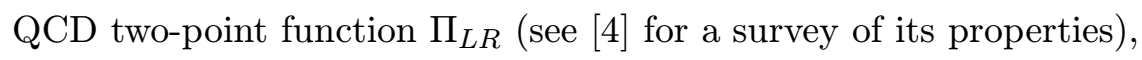

$$
\left\langle O_{1}\right\rangle=\frac{1}{2 i} \eta^{\mu \nu} \int \frac{d^{4} q}{(2 \pi)^{4}}\left(q_{\mu} q_{\nu}-q^{2} \eta_{\mu \nu}\right) \Pi_{L R}(q) .
$$

As it stands, this integral diverges and needs to be renormalized. For consistency, this should be done in the same scheme that was used in the computation of the Wilson coefficient $C_{7}(\mu, \kappa)$. As a first step, we regularize this integral in $D=4-\epsilon$ dimensions,

$$
\left\langle O_{1}\right\rangle^{\mathrm{reg}}=\frac{1-D}{32 \pi^{2}} \frac{\left(4 \pi \mu^{2}\right)^{\epsilon / 2}}{\Gamma(2-\epsilon / 2)} \int_{0}^{\infty} d Q^{2}\left(Q^{2}\right)^{1-\epsilon / 2}\left(-Q^{2} \Pi_{L R}\left(Q^{2}\right)\right)_{D} .
$$

The matching to the short-distance contributions contained in $C_{7}(\mu, \kappa)$ is controled by the OPE. In $D$ dimensions and in the large- $N_{C}$ limit, one has

$$
\lim _{Q^{2} \rightarrow \infty}(1-D)\left(-Q^{2} \Pi_{L R}\left(Q^{2}\right)\right)_{D}=-12 \pi^{2}\left(\frac{\alpha_{s}}{\pi}+\mathcal{O}\left(\alpha_{s}^{2}\right)\right)\left[1+(\kappa-2 / 3) \frac{\epsilon}{2}\right] \frac{\langle\bar{\psi} \psi\rangle^{2}}{Q^{4}},
$$

where $\kappa$ depends on the renormalization scheme. We find, in agreement with [ịin $], \kappa=-1 / 2$ in the NDR scheme, and $\kappa=+3 / 2$ in the HV scheme.

In order to obtain $\left.\left\langle O_{1}\right\rangle\right|_{\mathcal{O}\left(N_{C}\right)}$, we need to know $\left(-Q^{2} \Pi_{L R}\left(Q^{2}\right)\right)_{D}$ in the large- $N_{C}$ limit. But in this limit, $\left.\Pi_{L R}\left(Q^{2}\right)\right)_{D}$ is described by an infinite number of single poles, due 
to zero-width vector and axial resonances, and to the pion pole. Furthermore, one knows the asymptotic behaviour in $D$ dimensions, $\lim _{Q^{2} \rightarrow \infty}\left(-Q^{2} \Pi_{L R}\left(Q^{2}\right)\right)_{D} \sim 1 / Q^{6}$. Taken

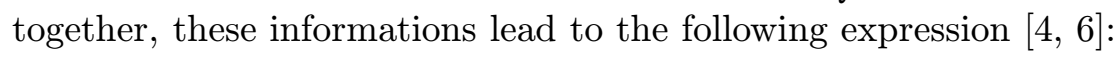

$$
\left(-Q^{2} \Pi_{L R}\left(Q^{2}\right)\right)_{D}=\sum_{A} \frac{f_{A}^{2} M_{A}^{6}}{Q^{2}\left(Q^{2}+M_{A}^{2}\right)}-\sum_{V} \frac{f_{V}^{2} M_{V}^{6}}{Q^{2}\left(Q^{2}+M_{V}^{2}\right)} .
$$

Then, after renormalization in the $\overline{\mathrm{MS}}$ scheme, one ends up with the formula

$$
\left.\left\langle O_{1}\right\rangle^{\overline{\mathrm{MS}}}(\mu, \kappa)\right|_{\mathcal{O}\left(N_{C}\right)}=-\frac{3}{32 \pi^{2}}\left[\sum_{A} f_{A}^{2} M_{A}^{6} \ln \frac{\Lambda^{2}}{M_{A}^{2}}-\sum_{V} f_{V}^{2} M_{V}^{6} \ln \frac{\Lambda^{2}}{M_{V}^{2}}\right],
$$

where the scale $\Lambda$ clearly shows the dependence with respect to the renormalization scale $\mu$ and to the renormalization scheme, $\Lambda^{2} \equiv \mu^{2} \exp (1 / 3+\kappa)$.

\section{Evaluation of $\left\langle\mathrm{O}_{2}\right\rangle$}

As mentioned earlier, the leading contribution to $\left\langle O_{2}\right\rangle$ in the $1 / N_{C}$ expansion is $\mathcal{O}\left(N_{C}^{2}\right)$ and corresponds to two disconnected flavour-singlet quark loops, $\left.\left\langle O_{2}\right\rangle\right|_{\mathcal{O}\left(N_{C}^{2}\right)}=\langle\bar{\psi} \psi\rangle^{2} / 4$. The correction to this result consists of the two previous quark loops connected by a planar gluonic configuration. This is a so-called Zweig-suppressed contribution, and is of order $\mathcal{O}\left(N_{C}^{0}\right)$. It is governed by a two-point function involving scalar and pseudoscalar densities,

$$
\left.\left\langle O_{2}\right\rangle\right|_{\mathcal{O}\left(N_{C}^{0}\right)}=\frac{1}{i} \int \frac{d^{4} q}{(2 \pi)^{4}} \Psi_{d s}\left(Q^{2}\right)
$$

with

$$
\Psi_{i j}\left(Q^{2}\right)=i \int d^{4} x e^{i q \cdot x}\left\langle 0\left|T\left\{\bar{q}_{i}(x) \frac{1+\gamma_{5}}{2} q_{i}(x) \bar{q}_{j}(0) \frac{1-\gamma_{5}}{2} q_{j}(0)\right\}\right| 0\right\rangle .
$$

As in the case of Eq. $\left(\overline{2}_{2} . \overline{1}_{1}\right)$, the integral in $\left(\overline{3}_{3} . \overline{1}_{1}\right)$ has to be regularized and renormalized in the same scheme as the one used for the short-distance calculation of the Wilson coefficients. One might think that the next step would proceed as in the case of the $\Pi_{L R}$ correlator, namely by setting up a large- $N_{C}$ ansatz for $\Psi_{d s}\left(Q^{2}\right)$, which correctly reproduces the QCD short-distance properties of this two-point function, which incidentally is also an order parameter of the $S U(3)_{L} \times S U(3)_{R}$ chiral symmetry. There is however little reason to expect such an approach to provide a realistic description of $\Psi_{d s}\left(Q^{2}\right)$, the reason being that it involves the singlet scalar and pseudoscalar channels, which are notoriously difficult to understand within a large- $N_{C}$ framework. For instance, a nonet of narrow scalar resonances is hard to identify, due to the mixing with scalar glueball states, or with a broad $\sigma$ resonance produced by strong final state interactions in the $J=0, I=0 \pi \pi$ channel $^{1}$, etc. In the singlet pseudoscalar channel, one encounters the $\eta^{\prime}$, which becomes a massless Goldstone boson in the large- $N_{C}$ limit, etc. All these effects are likely to be important,

\footnotetext{
${ }^{1}$ For a detailed review on the phenomenological situation in the scalar channel, see [i]j; for discussions on the Zweig rule violation in the scalar sector, see Refs. $\left[\mathbf{8}_{1}^{\prime \prime}, 9^{\prime}\right]$.
} 
although subleading in the large $N_{C}$ expansion. As an illustration, let us consider the contribution of the lowest pseudoscalar, flavour singlet meson to the integral (1י13),

$$
\left.\frac{1}{i} \int \frac{d^{4} q}{(2 \pi)^{4}} \Psi_{d s}\left(Q^{2}\right)\right|_{\eta^{[0)}} ^{\overline{\mathrm{MS}}}=-\frac{M_{\eta^{(0)}}^{2}}{16 \pi^{2} F_{0}^{2}} \frac{\langle\bar{\psi} \psi\rangle^{2}}{6}\left(\ln \frac{\mu^{2}}{M_{\eta^{(0)}}^{2}}+1\right) .
$$

Since $M_{\eta^{(0)}}^{2} \sim \mathcal{O}\left(1 / N_{C}\right)$, this contribution is indeed of order $N_{C}^{0}$. As compared to $\left.\left\langle O_{2}\right\rangle\right|_{\mathcal{O}\left(N_{C}^{2}\right)}$, the factor in front of the bracket containing the logarithm amounts to $\sim-0.5\langle\bar{\psi} \psi\rangle^{2} / 4$, a very large correction indeed, although the suppression factor is formally $1 / N_{C}^{2}$ !

In order to circumvent this difficulty, we come back to the short-distance behaviour (for $D=4$ ) in Eq. $(2.3)$, but without using the large- $N_{C}$ limit on the right-hand side,

$$
\lim _{Q^{2} \rightarrow \infty}\left(-Q^{2} \Pi_{L R}\left(Q^{2}\right)\right)_{D}=4 \pi^{2} \frac{\alpha_{s}}{\pi}\left[4\left(1+\xi(\kappa) \frac{\alpha_{s}}{\pi}+\cdots\right)\left\langle O_{2}\right\rangle+\left(\frac{2}{N_{C}}+\cdots\right)\left\langle O_{1}\right\rangle\right] / Q^{4} .
$$

The term proportional to $\left\langle O_{1}\right\rangle$ being small as compared to the other one (this can be checked a posteriori, using the numerical estimates given below), we neglect it and, using the large- $N_{C}$ ansatz (2) in 2 in order to compute the right-hand side, we arrive at

$$
\left\langle O_{2}\right\rangle^{\overline{\mathrm{MS}}}(\mu, \kappa) \mid=\frac{\sum_{A} f_{A}^{2} M_{A}^{6}-\sum_{V} f_{V}^{2} M_{V}^{6}}{16 \pi \alpha_{s}(\mu)\left[1+\xi(\kappa) \frac{\alpha_{s}(\mu)}{\pi}+\mathcal{O}\left(\alpha_{s}^{2}\right)\right]} .
$$

Notice that, due to the approximation that was made, the scheme dependence is no longer described in an exact manner. The only scheme dependence left over is burried in the coefficient $\xi(\kappa)$ of the $\mathcal{O}\left(\alpha_{s}^{2}\right)$ correction to the Wilson coefficient of the $\left\langle O_{2}\right\rangle$ term in $(3 . \overline{4})$, which was not known at the time Ref. [202] was written. In the meantime, it has been computed in Ref. [1] $\left.\left.{ }_{1}^{1} \overline{0}\right]_{1}^{\prime}\right]: \xi(\kappa=-1 / 2)=25 / 8$ (NDR), and $\xi(\kappa=3 / 2)=21 / 8(\mathrm{HV})$.

\section{Numerical values and concluding remarks}

We now turn to the numerical evaluations of the matrix elements $M_{7,8}=\left\langle(\pi \pi)_{2}\left|Q_{7,8}\right| K^{0}\right\rangle$ at order $\mathcal{O}\left(p^{0}\right)$, based on the expressions $\left(\overline{2} \cdot \overline{2}_{i}\right)$ and $\left(\overline{3} \cdot \overline{3} \overline{5}_{i}\right)$ for $\left\langle O_{1}\right\rangle$ and $\left\langle O_{2}\right\rangle$, with the respective sums over the vector and axial resonances restricted to the lowest lying state in each channel. For the various quantities that appear in these equations we use the values

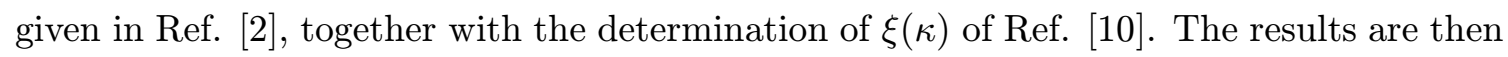
given in Table 1 below, where we also display other recent results for $M_{7,8}$.

With the $\mathcal{O}\left(\alpha_{s}^{2}\right)$ corrections included, our results are in good agreement with those obtained from a dispersive approach [i $100 ;$, and, in the case of $M_{8}$, numerically reproduce the same (mild) scheme dependence, despite of the approximation we have made. On the other hand, the differences with the lattice results [i] $\left.1_{1}^{1}\right]$ and with Ref. [i] Concerning the former, one should keep in mind that they were obtained in the quenched approximation, where the $\eta^{(0)}$ singlet is certainly very badly mistreated. As we have discussed, the $\eta^{(0)}$ contribution to $M_{8}$ could be important.

As far as our method is concerned, let us stress that it can be improved, by keeping several resonances in a given channel, provided additional information, for instance sub-

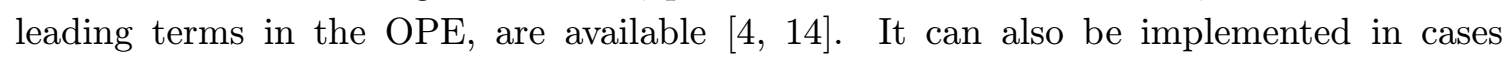




\begin{tabular}{|c|c|c|c|c|}
\hline Matrix Elements & $\bar{M}_{7}(\mathrm{NDR})$ & $\overline{M_{7}(\mathrm{HV})}$ & $\bar{M}_{8}(\mathrm{NDR})$ & $\overline{M_{8}(\mathrm{HV})}$ \\
\hline Ref. [1] $\left.\overline{1}_{1}^{1}\right]$ & $0.11 \pm 0.04$ & $0.18 \pm 0.06$ & $0.51 \pm 0.10$ & $0.62 \pm 0.12$ \\
\hline Refs. [1] $\overline{1} \overline{2} \overline{1}]$ & $0.26 \pm 0.03$ & $0.39 \pm 0.06$ & $1.2 \pm 0.5$ & $1.3 \pm 0.6$ \\
\hline Refs. $[\overline{\underline{n}}]$ & $0.22 \pm 0.05$ & & $1.3 \pm 0.3$ & \\
\hline Refs. [1] $[\overline{1} \overline{3}]$ & $0.35 \pm 0.10$ & & $2.7 \pm 0.6$ & \\
\hline Refs. $\left[\begin{array}{l}\overline{1} \\
1\end{array} \overline{0} \overline{0}\right]$ & $0.16 \pm 0.10$ & $0.49 \pm 0.07$ & $2.22 \pm 0.67$ & $2.46 \pm 0.70$ \\
\hline Ref. [2] and $\xi(\kappa)$ from Ref. [1] $\left.{ }_{1}^{1} \overline{0}\right]$ & $0.11 \pm 0.03$ & $0.67 \pm 0.20$ & $2.6 \pm 0.9$ & $2.9 \pm 1.1$ \\
\hline
\end{tabular}

Table 1: Summary of matrix elements $M_{7,8} \equiv\left\langle(\pi \pi)_{I=2}\left|Q_{7,8}\right| K^{0}\right\rangle_{(2 \mathrm{GeV})}$ using naive dimensional regularization (NDR) and the 't Hooft-Veltman scheme (HV), in units of $\mathrm{GeV}^{3}$.

where a dispersive approach is of little help in practice, for instance if three-point functions

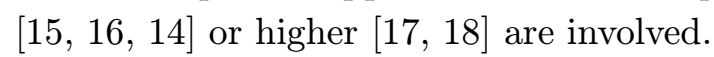

I wish to thank the organizers of this conference for making it an interesting and enjoyable meeting, as well as S. Peris and E. de Rafael for many discussions and a stimulating collaboration. Informative discussions with V. Cirigliano, L. Lellouch and A. Soni are also gratefully acknowledged.

\section{References}

[1] For a review, see e.g. A.J. Buras in Probing the standard model of particle interactions, Les Houches, 28 July - 5 Sept. 1997, R. Gupta et al. Eds., North Holland, 1999.

[2] M. Knecht, S. Peris and E. de Rafael, Phys. Lett. B 508, 117 (2001).

[3] M. Knecht, S. Peris and E. de Rafael, Phys. Lett. B 457, 227 (1999).

[4] M. Knecht and E. de Rafael, Phys. Lett. B 424, 335 (1998).

[5] J.F. Donoghue and E. Golowich, Phys. Lett. B 478, 172 (2000).

[6] M. Knecht, S. Peris and E. de Rafael, Phys. Lett. B 443, 255 (1998).

[7] D. Morgan, Nuovo Cim. A 107, 1883 (1994).

[8] B. Moussallam, Eur. Phys. J. C 14, 111 (2000); JHEP 0008, 005 (2000).

[9] S. Descotes and J. Stern, Phys. Rev. D 62, 054011 (2000); Phys. Lett. B 488, 274 (2000).

[10] V. Cirigliano, J. F. Donoghue, E. Golowich and K. Maltman, hep-ph/0109113.

[11] A. Donini et al., Phys. Lett. B 470, 233 (1999).

[12] J. Bijnens, E. Gamiz and J. Prades, JHEP 0110, 009 (2001).

[13] S. Narison, Nucl. Phys. B593, 3 (2001); see also Ref. [46] in E. de Rafael, hep-ph/0110195.

[14] M. Knecht and A. Nyffeler, Eur. Phys. J. C 21, 659 (2001).

[15] B. Moussallam, Nucl. Phys. B 504, 381 (1997).

[16] M. Knecht, S. Peris, M. Perrottet and E. de Rafael, Phys. Rev. Lett. 83, 5230 (1999).

[17] S. Peris and E. de Rafael, Phys. Lett. B 490, 213 (2000) and hep-ph/0006146.

[18] M. Knecht, in preparation. 